\title{
Spatially Guided Distractor Suppression during Visual Search
}

\author{
[Dobias Feldmann-Wüstefeld, ${ }^{1}{ }^{\circledR}$ Marina Weinberger, ${ }^{2}$ and ${ }^{\circledR}$ Edward Awh ${ }^{2,3}$ \\ ${ }^{1}$ Centre for Cognition and Perception, University of Southampton, Southampton, S017 1BJ, United Kingdom, ${ }^{2}$ Department of Psychology, \\ University of Chicago, Chicago, IL 60637, Illinois, and ${ }^{3}$ Institute for Mind and Biology, University of Chicago, Chicago, IL 60637, Illinois
}

Past work has demonstrated that active suppression of salient distractors is a critical part of visual selection. Evidence for goaldriven suppression includes below-baseline visual encoding at the position of salient distractors (Gaspelin and Luck, 2018) and neural signals such as the distractor positivity (Pd) that track how many distractors are presented in a given hemifield (FeldmannWüstefeld and Vogel, 2019). One basic question regarding distractor suppression is whether it is inherently spatial or nonspatial in character. Indeed, past work has shown that distractors evoke both spatial (Theeuwes, 1992) and nonspatial forms of interference (Folk and Remington, 1998), motivating a direct examination of whether space is integral to goal-driven distractor suppression. Here, we use behavioral and EEG data from adult humans (male and female) to provide clear evidence for a spatial gradient of suppression surrounding salient singleton distractors. Replicating past work, both reaction time and neural indices of target selection improved monotonically as the distance between target and distractor increased. Importantly, these target selection effects were paralleled by a monotonic decline in the amplitude of the $\mathrm{Pd}$, an electrophysiological index of distractor suppression. Moreover, multivariate analyses revealed spatially selective activity in the $\theta$-band that tracked the position of the target and, critically, revealed suppressed activity at spatial channels centered on distractor positions. Thus, goal-driven selection of relevant over irrelevant information benefits from a spatial gradient of suppression surrounding salient distractors.

Key words: attentional capture; EEG; multivariate models; Pd; suppression; visual attention

Significance Statement

Past work has shown that distractor suppression is an important part of goal-driven attentional selection, but has not yet revealed whether suppression is spatially directed. Using behavioral data, event-related potentials (ERPs) of the EEG signal [N2pc and distractor positivity (Pd) component], as well as a multivariate model of EEG data [channel tuning functions (CTF)], we show that suppression-related neural activity increases monotonically as the distance between targets and distractors decreases, and that spatially-selective activity in the $\theta$-band reveals depressed activity in spatial channels that index distractor positions. Thus, we provide robust evidence for spatially-guided distractor suppression, a result that has important implications for models of goal-driven attentional control.

\section{Introduction}

Visual attention allows us to cope with the vast amount of incoming information by selecting the most relevant subset of stimuli in our environment. Models of attentional control acknowledge multiple distinct influences on visual selection, including physical salience (Theeuwes, 2010), current goals (Folk and Remington, 2006), and learning experience (Awh et al., 2012; Hutchinson and Turk-Browne, 2012). Goal-driven

\footnotetext{
Received Sep. 15, 2020; revised Feb. 2, 2021; accepted Feb. 4, 2021.

Author contributions: T.F.-W. and E.A. designed research; M.W. performed research; T.F.-W. and M.W. analyzed data; T.F.-W. wrote the paper.

This work was supported by the National Institute of Mental Health Grant 2R01MH087214-06A1.

The authors declare no competing financial interests.

Correspondence should be addressed to Tobias Feldmann-Wüstefeld at tobias.fw@soton.ac.uk.

https://doi.org/10.1523/JNEUROSCI.2418-20.2021

Copyright $\odot 2021$ the authors
}

selection of targets among distractors has been shown to invoke a combination of target enhancement and distractor suppression. Relevant features can be enhanced to facilitate target processing (Desimone and Duncan, 1995; Wolfe, 2007), while irrelevant features can be suppressed below baseline to reduce distraction (Gaspelin et al., 2015; Chang and Egeth, 2019; FeldmannWüstefeld et al., 2020). Thus, recent models of visual attention implement enhancement and suppression as independent processes that resolve the competition of simultaneously presented stimuli for attentional resources (Liesefeld and Müller, 2019; Wyble et al., 2020).

Various EEG signals have been shown to track different aspects of selection. Task-relevant items elicit an N2pc component in the event-related potential (ERP) of the EEG signal, tracking the timing and probability of visual selection (Luck and Hillyard, 1994; Eimer, 1996). An established neural marker for distractor suppression is the distractor positivity $(\mathrm{Pd})$ component, 
typically elicited by irrelevant but salient and potentially distracting stimuli (Hickey et al., 2009; see also Feldmann-Wüstefeld and Schubö, 2013; Sawaki and Luck, 2013; Burra and Kerzel, 2014; Weaver et al., 2017; Gaspelin and Luck, 2018). Finally, inverted encoding models (IEMs) have been used to track attention deployment in the visual field with a high spatial resolution, revealing a gradient of enhancement surrounding an attended stimulus (Foster et al., 2016; Fahrenfort et al., 2017; Feldmann-Wüstefeld and Awh, 2020).

Here, we leveraged these EEG signals to examine whether distractor suppression during visual search is spatially guided. This question is motivated by prior work demonstrating both spatial and non-spatial forms of distractor interference. The neural ambiguity account posits increased interference for stimuli that are presented in the same receptive field (Desimone and Duncan, 1995; Luck et al., 1997). In line with this, distractor-induced interference monotonically increases when target and distractor are closer together (Kwak et al., 1991; Caputo and Guerra, 1998; Mounts, 2000; Theeuwes et al., 2004; Hickey and Theeuwes, 2011; Gaspar and Mcdonald, 2014). Other studies, however, found that salient distractors can also induce nonspatial filtering costs, i.e., interference independent of distractor position or target-distractor distance (Folk and Remington, 1998, 2006; Becker, 2007; Wykowska and Schubö, 2011). The robust evidence for both spatial and nonspatial forms of interference leaves open the question of whether distractor suppression is spatial in character, i.e., whether distractor interference is minimized via suppression of visual responses in specific locations. It is conceivable that the visual system resolves neural ambiguity by applying more suppression to distractors near the target at the cost of enhancing the target itself. Alternatively, distractor suppression may manage nonspatial forms of distractor interference and not vary with target-distractor distance accordingly.

While the contralateral nature of the Pd shows some spatial specificity, lateralized activity alone does not necessarily reflect a precise spatial representation. Thus, even with lateralized neural activity such as the $\mathrm{Pd}$ it is possible that suppression is not based on distractor position per se. For example, the Pd may index an attentional process that is lateralized, but for which the amount of suppression does not vary across positions within a hemifield. For the first time, the present work provides evidence for spatially-directed suppression of distractors by manipulating the distance between target and distractor stimuli, and observing the consequences for neural indices of suppression. The amplitude of the Pd component covaried with the distance between target and distractor, with monotonic increases in Pd amplitude as target and distractor were presented closer together. In addition, the Pd was eliminated when target and distractor shared a location, and spatial suppression would have also negatively impacted target processing. An Inverted Encoding Model (IEM) analysis of EEG oscillations (e.g., Foster et al, 2017) in the theta band revealed a reliable depression of spatial channel activity at the distractor position, providing converging evidence for a spatial gradient of suppression surrounding salient distractors.

\section{Materials and Methods}

\section{Overview}

First, in two behavioral pilots, we revised the additional singleton paradigm (Theeuwes, 1992, 2010) to statistically uncorrelate target and distractor location. In the additional singleton paradigm, a target is presented among distractors. Occasionally, one of the distractors is rendered salient by assigning it a unique color. Typically, target and salient distractor do not occur at the same location (for a review of paradigms in which target and distractor coincide, see Becker, 2007). In our revised additional singleton paradigm, we presented targets and distractors in the same location in $1 / \mathrm{d}$ of the distractor-present trials where $\mathrm{d}=$ display size. This was necessary to facilitate spatial modeling of target and distractor positions, and to avoid inducing strategies that affect attentional capture (Becker, 2007; Yantis and Egeth, 1999). Furthermore, it allowed us to examine neural indices of selection and suppression when the target and distractor shared the same location (compare Fockert et al., 2004; Hodsoll et al., 2009). In singleton-present trials of experiment $1 \mathrm{a}$, the target was red in $1 / \mathrm{d}$ of the trials while one of the nontargets was red in the remaining (d-1)/d of the trials. In singletonpresent trials of experiment $1 \mathrm{~b}$, an additional red ring was presented around the target in $1 / \mathrm{d}$ of the trials and around one of the nontargets in (d-1)/d of the trials. We then used the variant with the additional ring in two EEG experiments (experiments 2 and 3). In experiment 2, the distractor consistently had the same color throughout the experiment. In experiment 3, however, the distractor had a different color when it was presented in the target location, which provided an opportunity for observers to rely on color as a defining property of the target and more efficiently suppress the distractor when presented in a non-target position and more efficiently attend the target when coinciding with the distractor. As behavioral results were virtually identical in experiments 2 and 3 , we collapsed across these experiments to achieve a higher statistical power for EEG analyses.

\section{Participants}

In experiment 1a, 20 volunteers aged $19-31$ years $(M=21.5, S D=3.2)$, 13 female, all right-handed, participated. In experiment $1 \mathrm{~b}, 21$ volunteers aged 18-31 years $(M=23.5, S D=4.5), 11$ female, one left-handed participated. In experiments 2 and 3, 25 volunteers participated each, one participant from experiment 2 and two from experiment 3 were excluded because of excessive EEG artifacts (see criteria below). The remaining 24 participants in experiment 2 were aged $18-31$ years $(M=$ $22.8, S D=4.0)$, nine female, five left-handed. The remaining 23 participants in experiment 3 were aged $18-33$ years $(M=22.3, S D=3.8), 16$ female, one left-handed. Participants were naive to the paradigm and objectives of the study and were paid $\sim \$ 10$ (experiment 1 ) or $\sim \$ 15$ (experiments 2 and 3) per hour. All experiments were conducted with the written understanding and consent of each participant. The University of Chicago Institutional Review Board approved experimental procedures. Sample sizes were determined as 20 for behavioral experiments and 25 for EEG experiments, based on previous experiments using similar tasks.

\section{Apparatus}

Participants were seated in a dimly lit, electrically shielded and sound attenuated chamber. Participants responded with button presses on a standard keyboard that was placed in front of them. Stimuli were presented on an LCD computer screen (BenQ XL2430T; $120 \mathrm{~Hz}$ refresh rate; $61-\mathrm{cm}$ screen size in diameter; $1920 \times 1080$ pixels) placed at 75 $\mathrm{cm}$ distance from participants. An IBM-compatible computer (Dell Optiplex 9020) controlled stimulus presentation and response collection using MATLAB routines. In experiments 2 and 3, participants were seated with a chin-rest to allow for tracking gaze position.

\section{Stimuli}

All stimuli were presented on a gray background (RGB: 128-128-128). Search displays always showed six items presented equidistantly on an imaginary circle ( $3^{\circ}$ radius) around a black central fixation cross $\left(0.5^{\circ} \mathrm{di}-\right.$ ameter, RGB: 0-0-0; Fig. 1). Item positions were fixed with two items on the vertical midline $\left(0^{\circ}\right.$ eccentricity) and two items in each hemifield ( $2.6^{\circ}$ eccentricity). One of the items was a diamond ( $2.1^{\circ}$ diameter, 2 pixels line width), serving as a target stimulus. The other five items were nontarget circles $\left(1.6^{\circ}\right.$ diameter, same area as target, two pixels line width. In distractor-absent trials, all items were dark gray (RGB: 64-6464 ) and the target appeared equally often at one of the six positions. In distractor-present trials of experiment $1 \mathrm{a}$, one of the six items was red 
Distractor absent

A

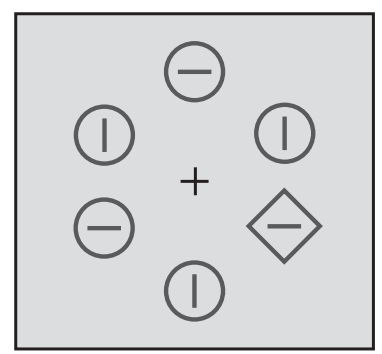

B
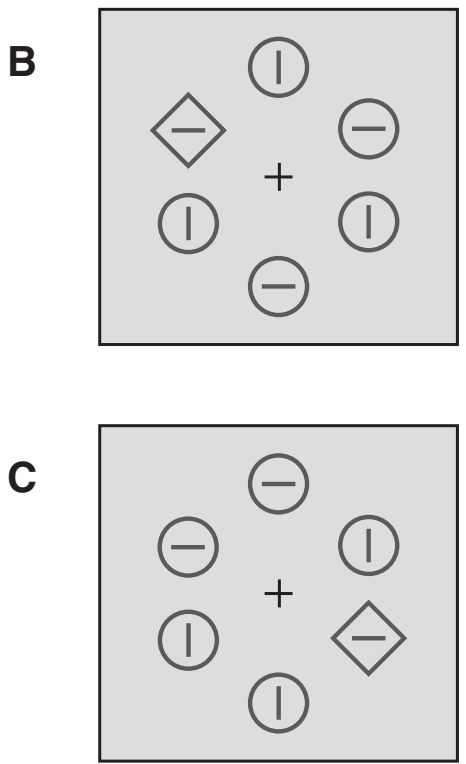

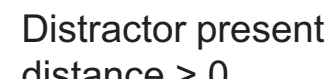

distance $>0$
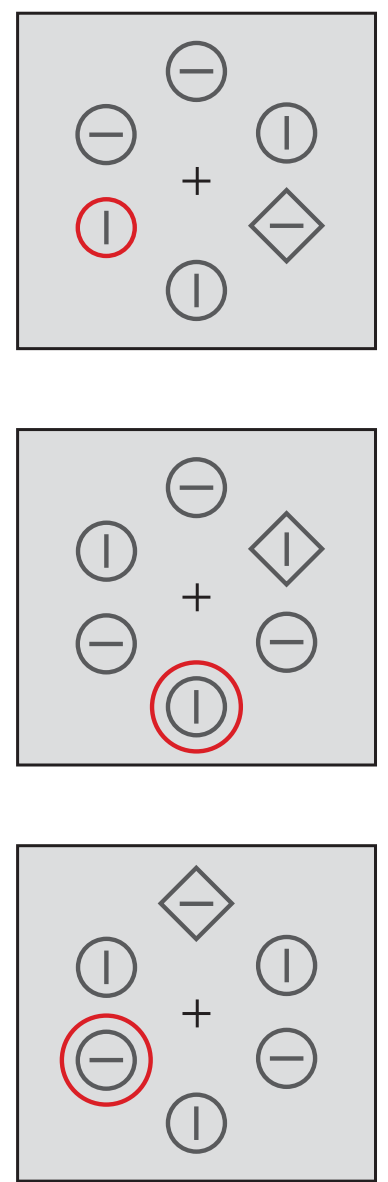

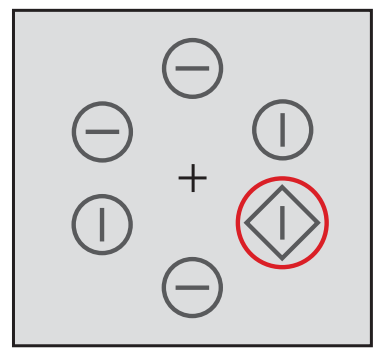

Distractor present

distance $=0$
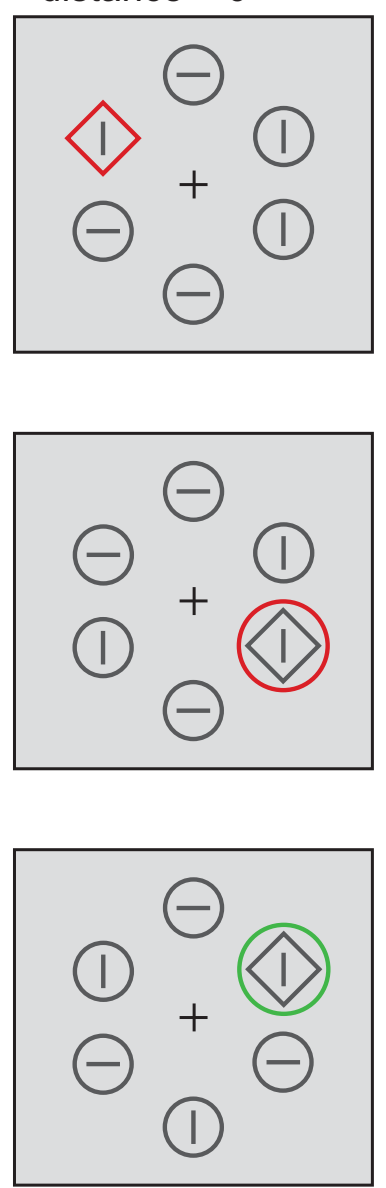

Figure 1. Visual search displays used for experiment $1 \mathrm{a}(\boldsymbol{A})$, experiments $1 \mathrm{~b}$ and $2(\boldsymbol{B})$, and experiment 3 ( $\boldsymbol{C}$. Participants had to find the diamond-shaped target and report the orientation of its embedded line with a key press. In half the trials, all items were gray (distractor absent trials). In the other half of the trials, a color singleton (red/green ring), serving as a distractor, was presented equally likely in one of the six positions. This means the singleton was in a distractor position in $5 / 6$ of singleton-present trials (distance $>0$ ) and in a target position in $1 / 6$ of singleton-present trials (distance $=0$ ). In experiment 3 , different color were used for distance $>0$ and distance $=0$ trials.

(RGB: 255-0-0). The singleton was presented equally often in each location, i.e., the target was a red diamond in $1 / 6$ of the distractor-present trials. In distractor-present trials of experiments $1 \mathrm{~b}$ and 2 , an additional empty red circle (2.4 ${ }^{\circ}$ diameter, 2 pixels line width, RGB: 255-0-0) appeared equally often around any one of the six items, i.e., around the target in $1 / 6$ of the distractor-present trials. In experiment 3 , the distractor had distinct colors for target versus nontarget positions (Fig. 1C). Distractor were red/green for nontarget locations and green/red for target locations (balanced across participants). The 36 combinations of target and distractor locations appeared equally often in distractor-present trials. Within each of the six items was a dark gray line that was either vertical or horizontal ( $0.75^{\circ}$ length, RGB: 64-64-64). Horizontal and vertical lines were used equally often for the target. In addition, in distractor-present trials in which the distractor was presented at a location different from the target location, the four combinations of horizontal and vertical lines were used equally often for the target and the distractor. The orientation of the lines within the remaining nontargets was randomly assigned with as many horizontal as vertical lines (or as close as possible).

\section{Procedure}

The trial procedure is shown in Figure 2. Each trial started with a display showing the fixation cross and no other objects for a duration of $500 \mathrm{~ms}$ (experiments $1 \mathrm{a}$ and $1 \mathrm{~b}$ ) or a randomly varying duration between 400 and $600 \mathrm{~ms}$ (experiments 2 and 3). Subsequently, the search display was shown for $200 \mathrm{~ms}$ in addition to the fixation cross. Finally, a response display with the fixation cross only was shown until participants responded or for a maximum of $1000 \mathrm{~ms}$. Participants were instructed to report the orientation (horizontal or vertical) of the line within the diamond-shaped target by pressing a key corresponding to each orientation (key assignments were counterbalanced across participants). Their responses were limited to a time window of $1200 \mathrm{~ms}$ (search display + response display), and responses made after this period were not counted. If participants did not respond within this time window, a message reading "too slow" was shown for $500 \mathrm{~ms}$. After this message, or after participants responded, a blank screen was shown for a duration of $1000 \mathrm{~ms}$ (experiments $1 \mathrm{a}$ and $\mathrm{lb}$ ) or a randomly varying duration between 800 and $1200 \mathrm{~ms}$ (experiments 2 and 3). To optimally prepare participants to avoid eye movements which would abort a trial (see below), a preparation display was shown before the start of a trial in experiments 2 and 3 . The preparation display showed a single black fixation dot in the center of the screen $\left(0.25^{\circ}\right.$ diameter, RGB: 0-0-0). Participants were told to fixate the dot and not move their eyes or blink until the end of a trial. After subjects pressed the spacebar, the trial started with showing the fixation cross.

Participants were given the opportunity to practice the task before the experiment began. Once participants reached $75 \%$ accuracy and felt comfortable doing the task, they began the experiment.

Experiments 1a and $1 \mathrm{~b}$ consisted of 24 blocks of 36 trials (864 trials in total). Half the trials (432) were singleton-absent, the other half (432) were singleton-present. Experiments 2 and 3 consisted of 28 blocks of 72 trials (2016 trials in total). Half the trials (1008) were distractor-absent, 
Ready Display

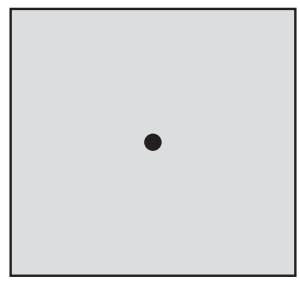

[until button press]
Fixation Cross

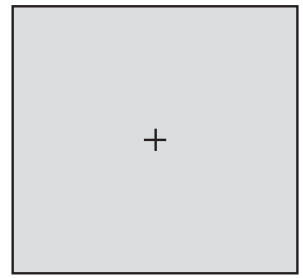

$400-600 \mathrm{~ms}$
Search Display

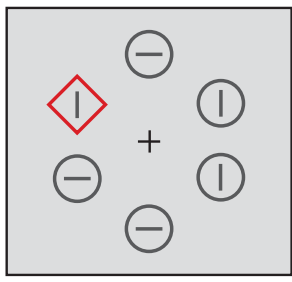

$200 \mathrm{~ms}$
Response Display

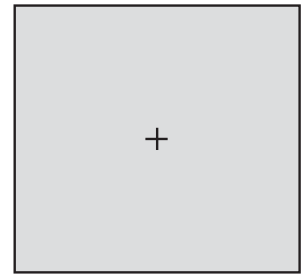

$1000 \mathrm{~ms}$

Figure 2. Trial procedure. Each trial started with a ready display. Participants had to fixate the central dot and press a key when ready. Upon key press a fixation cross followed for $400-$ $600 \mathrm{~ms}(500 \mathrm{~ms}$ in experiment 1) before the search display was presented for $200 \mathrm{~ms}$. The subsequent response display only showed a fixation cross. Participants had to respond during the search display or response display presentation (i.e., within $1200 \mathrm{~ms}$ ). The response display was followed by an intertrial interval showing a blank screen for $1000 \mathrm{~ms}$ (experiment 1 ) or 800 $1200 \mathrm{~ms}$ (experiments 2 and 3). The ready display was not used in experiment 1.

the other half (1008) were distractor-present. All trials were presented in random order across an entire experiment. After each block, participants were given feedback about their performance (percent correct, number of slow trials, and if a new "high score" was achieved for accuracy during that block). Following feedback, there was a mandatory 10-s break after which participants could choose to either immediately continue to the next block or to extend their break.

\section{Eye tracking (experiments 2 and 3 only)}

In experiments 2 and 3 , gaze position was tracked at a sampling rate of $1000 \mathrm{~Hz}$ for both eyes with an EyeLink 1000+ eye tracker (SR Research Ltd.). A direct gaze violation feedback procedure was applied from halfway through the fixation display until participants responded or the response time (RT) window ended. The trial was aborted if the participants' gaze was not within $1.5^{\circ}$ of the center of the fixation cross during this time or if they blinked. A message (either "eye movement" or "blink") was presented on the screen, and then the next trial began with the new preparation display. Each aborted trial was added back into the sequence of upcoming trials, extending the experiment by one trial, and the trial sequence was re-shuffled so as to make the aborted trial's reappearance unpredictable. The mean number of total trials (including repeated trials) was $2140(\mathrm{SD}=126)$ in experiment 2 and $2224(\mathrm{SD}=171)$ in experiment 3.

\section{EEG recording (experiments 2 and 3 only)}

EEG was recorded using $\mathrm{Ag}$ - $\mathrm{AgCl}$ active electrodes (Brain-Products actiCAP) from 32 scalp sites (according to the International 10/20 System: FP 1/2, F7/8, F3/4, Fz, FC5/6, FC1/2, C3/4, Cz, TP9/10, CP5/6, $\mathrm{CP} 1 / 2, \mathrm{P} 7 / 8, \mathrm{P} 3 / 4, \mathrm{PO} 7 / 8, \mathrm{PO} 3 / 4, \mathrm{Pz}, \mathrm{O} 1 / 2, \mathrm{Oz})$. FPz served as the ground electrode and all electrodes were referenced online to TP10 and re-referenced off-line to the average of all electrodes. Incoming data were filtered (low cutoff $=0.01 \mathrm{~Hz}$, high cutoff $=250 \mathrm{~Hz}$, slope from lowto high-cutoff $=12 \mathrm{~dB} /$ octave) and recorded with a $1000 \mathrm{~Hz}$ sampling rate. Impedances were kept below $10 \mathrm{k} \Omega$. Horizontal and vertical electrooculograms (EOGs) were recorded with passive electrodes bipolarly $\sim 1 \mathrm{~cm}$ from the outer canthi of the eyes and from $1 \mathrm{~cm}$ above and $2 \mathrm{~cm}$ below the observers' right eye, respectively.

\section{Design and data analysis}

There were two main conditions: distractor-absent and distractor-present trials. Since trials with coinciding target and distractor constitute a special case, three conditions were considered: distractor-absent, distractor-target distance $=0$ (from here on "Dist $=0$ "), and distractor-target distance $>0$ (from here on "Dist $>0$ "). Accordingly, mean RTs and error rates were forwarded to a one-way ANOVA for repeated measures with three factor levels. In addition, RTs in distractor-present trials were analyzed as a function of distance between target and distractor. As there were six positions and target and distractor could be presented at the same location, there was a total of four distances used $(0,1,2,3)$. For all
RT analyses, only correct trials were used. For all experiments, Greenhouse-Geisser correction was applied where appropriate. Effect sizes are reported as partial $\eta^{2}$ for ANOVAs and Cohen's $d$ for $t$ tests.

Experiments 2 and 3 had the same conditions regarding behavioral measures as experiment 1 . With regards to ERPs, the lateralization of stimuli plays a crucial role (Woodman and Luck, 2003; Hickey et al., 2009). For ERP analyses, we focused on trials in which either the target was presented laterally and the distractor was on the vertical midline (or absent) or the distractor was presented laterally and the target was on the vertical midline. These trials do not confound target and distractor activity in the lateralized ERP and allow the disentanglement of target and distractor processing. The ERP was analyzed separately for targetdistractor distances 0,1 , or 2 . Note that the eccentricity for positions at distance 1 and 2 was identical; this is important because eccentricity can affect the amplitude of attention-related components (Papaioannou and Luck, 2020). Six conditions resulted for ERP analyses in both experiments 2 and 3: (1) target lateral, singleton absent; (2) target and singleton at same lateral location, distance 0 ; (3) target lateral, singleton midline, distance 1 ; (4) target lateral, singleton midline, distance 2; (5) singleton lateral, target midline, distance 1 ; (6) singleton lateral, target midline, distance 2 .

\section{EEG data analysis (experiments 2 and 3 only)}

EEG was averaged off-line over a 700 -ms epoch including a 200 -ms prestimulus baseline with epochs time-locked to search display onset. Trials with incorrect or no responses were excluded. Trials with eye-related artifacts from -200 to $600 \mathrm{~ms}$ were excluded from the analysis (experiment $2: \mathrm{M}=18.0 \%, \mathrm{SD}=11.3 \%$; experiment $3: \mathrm{M}=23.8 \%, \mathrm{SD}=12.8 \%$ ). To identify eye-related artifacts, eye tracking data were first baselined identically to EEG data (i.e., subtraction of the mean amplitude of $\mathrm{x}$ and y coordinates for the time from -200 to $0 \mathrm{~ms}$ ). Then, the Euclidian distance from the fixation cross was calculated from baselined data. We identified saccades with a step criterion of $0.5^{\circ}$ (comparing the mean position in the first half of a $50-\mathrm{ms}$ window with the mean position in the second half of a 50-ms window; window moved in 20-ms steps). We identified drifts by eye tracking data indicating a distance from the fixation $>1.2^{\circ}$. To minimize false alarms because of noise in the data, both eyes had to indicate an eye-related artifact for a trial to be excluded from analysis. For one participant, eye tracking data of sufficient quality was not available. For this participant, the same procedure as for eye tracking based artifact rejection was used with these criteria: $25 \mu \mathrm{V}$ for hEOG (difference left and right channel), $100 \mu \mathrm{V}$ for vEOG (difference upper and lower channel). Participants with fewer than 1000 trials were excluded from analyses (experiment 2: $n=1$; experiment 3: $n=2$ ).

\section{ERPs}

For all ERP-relevant conditions (see above), mean contralateral and ipsilateral ERP activity was calculated for each participant for an electrode pool of the three symmetric sites PO7/PO8, P7/8, PO3/4. For statistical analyses, the lateralized waveform (contra- minus ipsilateral activity) 
was used. The time windows for statistical analyses were determined in a data-driven way. For each component of interest, a 100-ms time window was determined around the peak of the component collapsed across conditions. The mean amplitude of the respective component was calculated for these time windows and forwarded to ANOVAs for repeated measures. The N2pc was compared for trials with lateral targets and no distractor, distractors at distance 1 , and distractors at distance 2. The posterior positivity contralateral $(\mathrm{Ppc})$, distractor-N2pc, and $\mathrm{Pd}$ were compared for trials with lateral distractors and targets in the same position as the distractor, for targets at distance 1 , and for targets at distance 2 .

\section{IEM}

While ERPs allow the measurement of visual priority on the coarse scale of left versus right hemifield, IEMs can be used to reconstruct spatially selective channel tuning functions (CTFs) from the topographic distribution of EEG activity across electrodes (Ester et al., 2015; Foster et al., 2016; Fahrenfort et al., 2017). We used an IEM that assumes that power measured at each electrode reflects the weighted sum of six spatial channels (representing neuronal populations), each tuned for one of the six item positions. The IEM was run on evoked power (activity phaselocked to stimulus onset). As both $\alpha$ band (Sauseng et al., 2005; Foster et al., 2016) as well as $\theta$-band (Dowdall et al., 2012; Harris et al., 2017) frequencies have shown to track attention deployment, the IEM was run on these frequency bands. To isolate frequency-specific activity, raw EEG segments were bandpass filtered for the $\alpha$ frequency range $(8-12 \mathrm{~Hz})$ as well as for the $\theta$ frequency rage $(4-8 \mathrm{~Hz})$ using a two-way least-squares finite-impulse-response filter ("eegfilt.m" from EEGLAB Toolbox) and then Hilbert-transformed. The complex analytic signal was first averaged across trials, and then the complex magnitude of the averaged analytic signal was squared. In a training stage, segmented, filtered waveforms from distractor-absent trials (equaled for target location) were used to estimate the weights for a model of the target's spatial position in a leastsquares estimation. Each artifact-free trial was assigned to one of two bins (bin assignment had 1000 iterations to allow for cross-validation). Electrodes with $>50$ trials of noise were excluded from the analysis to minimize the loss of trials. In the test stage, the model was inverted to transform the remaining half of trials (equaled for positions) into estimated channel responses, using the previously determined weights, for each of the two bins. Channel responses were averaged across bins and bin assignment iterations. Testing was done twice using the same trials; once using the target position labels, once using the distractor position labels. This means that a common set of training data were used to estimate the channel responses for targets and singletons separately, but in the same trials. This procedure precludes the possibility that any observed effects of attention deployment are because of differences that arose during training or because of using distinct "basis sets" for the two conditions. The six channel response functions were shifted to a common center and averaged to obtain the CTF. The slope of the CTFs (estimated by linear regression computed for each time point) was used as a metric to compare attention deployment toward target and distractor. The slope was compared between channels tested on target versus distractor position with $t$ test for dependent measures for each time point between 0 and $500 \mathrm{~ms}$. Only clusters of significance in which 50 consecutive data points (i.e., $50 \mathrm{~ms}$ ) have to show a $p<0.05$ were considered reliable.

\section{Results}

\section{Experiment 1a}

Distractor-absent trials were fastest $(555 \mathrm{~ms})$, followed by Dist $=0$ trials $(566 \mathrm{~ms})$ and Dist $>0$ trials $(582 \mathrm{~ms}$; Fig. $3 A$, upper panel). This was confirmed by a reliable main effect of trial type, $F_{(2,38)}=23.7, p<0.001, \eta^{2}=0.56$. All direct comparisons were significant (all $p \leq 0.012$ ). Trial type also affected accuracy $\left(\mathrm{M}_{\text {absent }}=90.7 \%, \mathrm{M}_{\text {dist }=0}=90.8 \%, \mathrm{M}_{\text {dist }>0}=88.6 \%\right), F_{(2,38)}=3.3$, $p=0.047, \eta^{2}=0.15$. Direct comparisons show reliable differences between distractor-absent and Dist $>0$ trials $(p=0.033)$, but not between any other condition (both $p \geq 0.053$ ).
In distractor-present trials, RT was fastest for Dist $=0$ trials. For Dist $=1$, RT was slowest, with monotonically decreasing RTs for increasing distances (Fig. $3 A$, lower panel). This was confirmed by a reliable main effect of distance, $F_{(3,57)}=13.7$, $p<0.001, \eta^{2}=0.42$. All direct comparisons between distances were reliable (all $p \leq 0.020$ ) except for distance 0 and 3 $(p=0.382)$. The distance between target and distractor also affected accuracy $\left(\mathrm{M}_{0}=90.8 \%, \mathrm{M}_{1}=87.9 \%, \mathrm{M}_{2}=88.6 \%, \mathrm{M}_{3}=\right.$ $90.0 \%), F_{(3,57)}=2.9, p=0.041, \eta^{2}=0.13$. Only the direct comparisons between distance 0 and 1 was reliable $(p=0.030)$, none of the other direct comparisons were reliable (both $p \geq 0.051$ ).

In compatible trials (target and distractor line of same orientation) RTs were equally long $(\mathrm{M}=581 \mathrm{~ms})$ as in incompatible trials $(\mathrm{M}=584 \mathrm{~ms}), t_{(19)}=0.7, p=0.236, d=0.04$. Accuracy, however, was higher in compatible $(\mathrm{M}=89.5 \%)$ than in incompatible trials $(\mathrm{M}=87.7 \%), t_{(19)}=1.9, p=0.040, d=0.18$.

In sum, the results show best performance in distractor absent trials and a spatial gradient of interference in distractor present trials. RT rose monotonically as the distance between target and distractor declined. The compatibility effect is further evidence for spatial interference as it suggests that distractors were processed to some extent (Becker, 2007).

\section{Experiment 1b}

Similar to experiment $1 \mathrm{a}$, distractor-absent trials were fastest $(587 \mathrm{~ms})$, followed by Dist $=0$ trials $(618 \mathrm{~ms})$ and Dist $>0$ trials (633 ms; Fig. 3B, upper panel). This was confirmed by a reliable main effect of trial type, $F_{(2,40)}=33.8, p<0.001, \eta^{2}=0.63$. All direct comparisons were significant (all $p \leq 0.042$ ). Trial type also affected accuracy $\left(\mathrm{M}_{\text {absent }}=93.3 \%, \mathrm{M}_{\text {dist }=0}=91.8 \%, \mathrm{M}_{\text {dist }>0}=\right.$ $90.7 \%), F_{(2,40)}=4.5, p=0.017, \eta^{2}=0.18$. Direct comparisons show reliable differences between distractor-absent and Dist $>$ 0 trials $(p=0.006)$, but not between any other condition (both $p \geq 0.073)$.

Similar to experiment 1a, RT was fastest for Dist $=0$ trials and slowest for Dist $=1$, with monotonically decreasing RTs for increasing distances (Fig. 3B, lower panel). This was confirmed by a reliable main effect of distance, $F_{(3,60)}=18.7, p<0.001, \eta^{2}=$ 0.48 . All direct comparisons between distances were reliable (all $p \leq 0.025)$ except for distances 0 and $3(p=0.553)$ and distances 0 and $2(p=0.511)$. The distance between target and distractor did not affect accuracy $\left(\mathrm{M}_{0}=91.8 \%, \mathrm{M}_{1}=89.7 \%, \mathrm{M}_{2}=91.4 \%\right.$, $\left.\mathrm{M}_{3}=91.3 \%\right), F_{(3,60)}=1.6, p=0.196, \eta^{2}=0.08$. No direct comparisons between distances were reliable (all $p \geq 0.097$ ).

In compatible trials, RTs were shorter $(\mathrm{M}=625 \mathrm{~ms})$ than in incompatible trials, $(\mathrm{M}=641 \mathrm{~ms}), t_{(19)}=3.8, p<0.001, d=0.20$. Analogously, accuracy was higher in compatible $(M=92.3 \%)$ than in incompatible trials $(M=89.1 \%), t_{(19)}=3.0, p=0.003$, $d=0.53$. In sum, the spatial gradient of interference as well as the compatibility effect from experiment 1 a were replicated.

\section{Behavioral results experiment 2}

Similar to experiment 1, distractor-absent trials were fastest $(558 \mathrm{~ms})$, followed by Dist $=0$ trials $(583 \mathrm{~ms})$ and Dist $>0$ trials (609 ms; Fig. 3C, upper panel). This was confirmed by a reliable main effect of trial type, $F_{(2,46)}=33.7, p<0.001, \eta^{2}=0.59$. All direct comparisons were significant (all $p \leq 0.004$ ). Trial type also affected accuracy $\left(\mathrm{M}_{\text {absent }}=92.1 \%, \mathrm{M}_{\text {dist }=0}=91.1 \%, \mathrm{M}_{\text {dist }>0}\right.$ $=88.9 \%), F_{(2,46)}=15.1, p<0.001, \eta^{2}=0.40$. Direct comparisons show reliable differences between distractor-absent and Dist $>0$ trials and between distractor-absent and Dist $=0$ trials (both $p \leq 0.007)$, but not between Dist $=0$ and Dist $>0(p=0.073)$. 

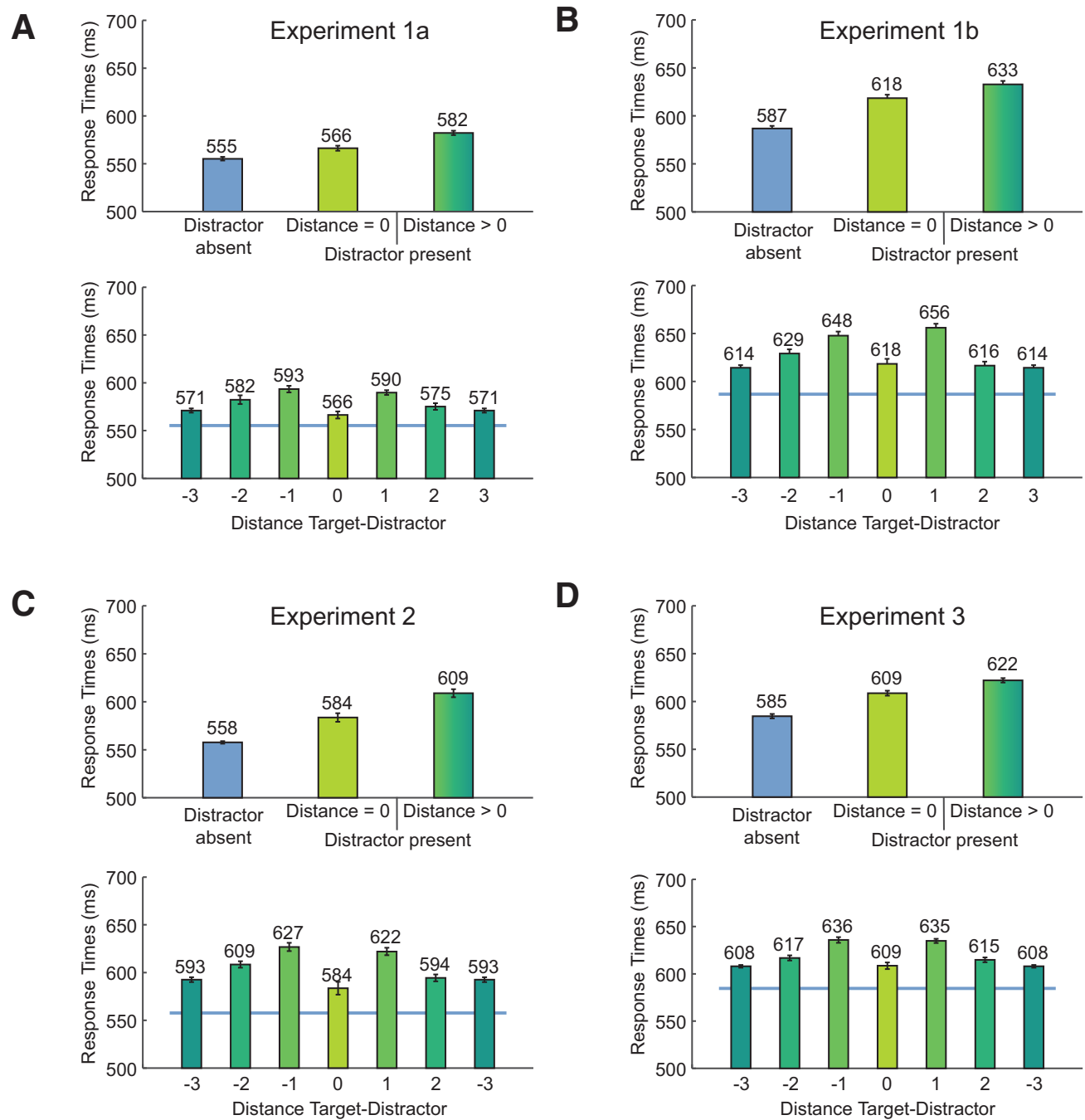

Figure 3. Behavioral results in experiments $1 \mathrm{a}(\boldsymbol{A}), 1 \mathrm{~b}(\boldsymbol{B}), 2(\boldsymbol{C})$, and $3(\boldsymbol{D})$. The upper panels shows RT as a function of trial type (distractor absent, distractor-target distance $=0$, distractor-target distance $>0$ ). The lower panels show RT as a function of distance between target and distractor in distractor-present trials (blue line depicts RT in distractor-absent trials for comparison). Negative numbers represent counterclockwise distances, positive numbers clockwise distances. Distance 0 refers to trials in which target and distractor appeared at the same location (experiments 1b, 2 and 3) or the target was the color singleton itself (experiment 1a). Error bars denote SEMs, corrected for within-subjects variability (Cousineau, 2005).

Similar to experiment 1 , RT was fastest for Dist $=0$ trials and slowest for Dist $=1$, with monotonically decreasing RTs for increasing distances (Fig. 3C, lower panel). This was confirmed by a reliable main effect of distance, $F_{(3,69)}=13.4, p<0.001, \eta^{2}=0.37$. All pairwise comparisons were significant (all $p \leq 0.037$ ) except the comparison between distance 0 and $3(p=0.237)$. The distance between target and distractor also affected accuracy $\left(\mathrm{M}_{0}=91.1 \%\right.$, $\left.\mathrm{M}_{1}=87.2 \%, \mathrm{M}_{2}=89.7 \%, \mathrm{M}_{3}=90.4 \%\right), F_{(3,69)}=11.8, p<0.001$, $\eta^{2}=0.34$. Pairwise comparisons were significant (all $p<0.001$ ) except for distance 0 versus 3,0 versus 2 , and 2 versus 3 ( $p \geq 0.067$ ).

In compatible trials (target and distractor line of same orientation) RTs were shorter $(\mathrm{M}=596 \mathrm{~ms})$ than in incompatible trials, $(\mathrm{M}=622 \mathrm{~ms}), t_{(23)}=8.3, p<0.001, d=0.48$. Analogously, accuracy was higher in compatible trials $(\mathrm{M}=91.7 \%)$ than in incompatible $(\mathrm{M}=86.1 \%)$ trials, $t_{(23)}=5.2, p<0.001, d=0.77$. In sum, the spatial gradient of interference as well as the compatibility effect from experiment 1 were replicated.

\section{Behavioral results experiment 3}

Similar to experiments 1 and 2, distractor-absent trials were fastest $(585 \mathrm{~ms})$, followed by Dist $=0$ trials $(609 \mathrm{~ms})$ and Dist $>0$ trials $(622 \mathrm{~ms}$; Fig. $3 D$, upper panel). This was confirmed by a reliable main effect of trial type, $F_{(2,44)}=41.9, p<0.001, \eta^{2}=0.66$. All direct comparisons were significant (all $p \leq 0.005$ ). Trial type also affected accuracy $\left(\mathrm{M}_{\text {absent }}=92.7 \%, \mathrm{M}_{\text {dist }=0}=91.5 \%, \mathrm{M}_{\text {dist }>0}=\right.$ $90.3 \%), F_{(2,44)}=9.1, p=0.001, \eta^{2}=0.29$. Direct comparisons show reliable differences between distractor-absent and Dist $>0$ trials and between distractor-absent and Dist $=0$ trials (both $p \leq 0.021$ ), but not between Dist $=0$ and Dist $>0(p=0.068)$.

Similar to experiments 1 and 2, RT was fastest for Dist $=0$ trials and slowest for Dist $=1$, with monotonically decreasing RTs for increasing distances (Fig. 3D, lower panel). This was confirmed by a reliable main effect of distance, $F_{(3,66)}=25.7$, $p<0.001, \eta^{2}=0.54$. All pairwise comparisons were significant (all $p \leq 0.002$ ) except for the comparison distance 0 versus 3 and 0 versus 2 (both $p \geq 0.116$ ). The distance between target and distractor also affected accuracy $\left(\mathrm{M}_{0}=91.5 \%, \mathrm{M}_{1}=89.1 \%, \mathrm{M}_{2}=\right.$ $\left.91.0 \%, \mathrm{M}_{3}=91.6 \%\right), F_{(3,66)}=6.1, p=0.004, \eta^{2}=0.22$. Pairwise comparisons were significant (all $p<0.005$ except for distance 0 versus 2,0 versus 3 , and 2 versus 3 (all $p \geq 0.285$ ).

In compatible trials RTs were shorter $(\mathrm{M}=615 \mathrm{~ms})$ than in incompatible trials $(M=629 \mathrm{~ms}), t_{(22)}=5.5, p<0.001, d=0.25$. Similarly, accuracy was higher $(\mathrm{M}=92.3 \%)$ in compatible than in incompatible trials $(\mathrm{M}=88.4 \%), t_{(22)}=6.1, p<0.001, d=0.68$. 
In sum, the spatial gradient of interference as well as the compatibility effect from experiments 1 and 2 were replicated. Of particular interest for analyzing EEG data are the identical pattern of behavioral results in experiments 2 and 3.

\section{Comparison experiments 2 and 3}

The similarity of behavioral results in experiments 2 and 3 was first confirmed with a ANOVA for repeated measures using the within-subjects factor trial type (distractor-absent, Dist $=0$, Dist $>0$ ) and the between-subjects factor experiment (2 vs 3 ), which showed neither a main effect of experiment, $F_{(1,45)}=2.0$, $p=0.162, \eta^{2}=0.04$, nor an interaction of trial type and experiment, $F_{(2,90)}=1.9, p=0.163, \eta^{2}=0.04$. Second, an ANOVA for repeated measures was run using the within-subjects factor distance $(0,1,2,3)$ and the between-subjects factor experiment (2 vs 3 ). Neither experiment, $F_{(1,45)}=1.1, p=0.303, \eta^{2}=0.02$ nor the interaction of experiment and distance, $F_{(3,135)}=1.2$, $p=0.293, \eta^{2}=0.03$ showed reliable effects. Thus, our results indicate that using a distinct color for distance-zero trials where targets and distractor positions coincide does not yield an attention deployment different from using the same color for distance-zero and other distance $>0$ trials.

In sum, the behavioral results from experiments 1-3 revealed both spatial and nonspatial filtering costs. Faster RTs with increasing distance between target and distractor indicate a spatial gradient of interference. Further evidence for spatial interference comes from the finding that distractors appearing at the same location as the target yield faster RTs than distractors appearing at different locations; when target and distractors spatially coincided, more pronounced attention deployment was observed. Moreover, compatibility effects support the notion of spatial interference; faster responses for identical target and distractor identity suggest that attention is deployed toward the distractor before the target in a subset of trials. Evidence for nonspatial interference comes from the comparison of distractor-absent trials and trials in which target and distractor coincide. As trials without any distractor yielded faster RTs than trials in which target and distractor appear at the same location, our results suggest that the mere presence of a distractors results in filtering costs that are not well explained by spatial capture. A pure spatial account of attentional capture (Theeuwes, 2010) would have predicted that target and distractor at the same location result in the most efficient attention deployment as two singletons coincide. Even when top-down control is facilitated by presenting the additional singleton in a distinct color when presented at the target location (experiment 3), this effect was replicated, demonstrating robust nonspatial filtering costs.

\section{ERP results experiments 2 and 3}

As behavioral results were virtually identical in experiments 2 and 3, we collapsed the EEG data from experiments 2 and 3 to achieve a higher statistical power. ERPs are shown in Figure 4.

$\mathrm{N} 2 p c$ in target-lateral trials (223-322 $\mathrm{ms}$ )

The N2pc was significantly different from zero in all conditions (all $p<0.001$ ). The N2pc was larger for distractors at distance 2 $(\mathrm{M}=-0.85)$ than at distance $1(\mathrm{M}=-0.56), t_{(46)}=2.6, p=0.012$, $d=0.39$, suggesting more attention deployment toward the target when the distractor was further away. The N2pc was also larger in distractor-absent trials $(\mathrm{M}=-0.93)$ than for distractors at distance $1, t_{(46)}=3.2, p=0.002, d=0.51$, but not larger than for distractors at distance $2, t_{(46)}=1.0, p=0.327, d=0.11$. This suggests that a target alone was attended more strongly than a target with a nearby distractor, but not more than a target with a further away distractor. The direct comparisons of target-N2pc amplitudes between trial types were confirmed with a significant oneway ANOVA, $F_{(2,92)}=7.0, p=0.001, \eta^{2}=0.13$.

\section{N2pc in distractor-lateral trials (175-274 ms)}

The N2pc was significantly different from zero in all conditions (all $p<0.001$ ). Distractor-N2pc amplitude was equally large for targets at distance 2 and at distance $1, t_{(46)}=1.7, p=0.095, d=$ 0.16 , suggesting that distractors capture attention equally strongly regardless of the distance to a target. The N2pc elicited by target and distractor in coinciding positions $(\mathrm{M}=-1.43)$ was larger than the distractor-N2pc for targets at distance $1(\mathrm{M}=-0.69), t_{(46)}=6.2$, $p<0.001, d=0.68$, and for targets at distance $2(\mathrm{M}=-0.85)$, $t_{(46)}=5.6, p<0.001, d=0.49$. In addition, the N2pc was larger in trials with coinciding target and distractor positions than in distractor absent trials, $t_{(46)}=4.1, p<0.001, d=0.47$. This indicates that attention is most strongly deployed to a location shared by target and distractor. The direct comparisons of distractor-N2pc amplitudes between trial types were confirmed with a significant one-way ANOVA, $F_{(2,92)}=26.7, p<0.001, \eta^{2}=0.37$.

\section{Pd (255-354 ms)}

The Pd was significantly different from zero for distances 1 and 2 (both $p<0.001$ ), but not for coinciding target and distractor positions $(p=0.310)$.

The effect of distance on Pd amplitude was the opposite of the effect on the target-N2pc: Pd amplitude was larger for targets at distance 1 than at distance $2, t_{(46)}=3.6, p=0.001, d=0.44$. At the same time, the Pd elicited by target and distractor in coinciding positions $(\mathrm{M}=-0.20)$ was smaller than the $\mathrm{Pd}$ for targets at distance $1(\mathrm{M}=1.24), t_{(46)}=8.7, p<0.001, d=1.18$, and for targets at distance $2(\mathrm{M}=0.82), t_{(46)}=6.2, p<0.001, d=0.92$. The direct comparisons of $\mathrm{Pd}$ amplitudes between trial types were confirmed with a significant one-way ANOVA, $F_{(2,92)}=48.0$, $p<0.001, \eta^{2}=0.51$.

\section{Exploratory analysis: Ppc (79-178 ms)}

The $\mathrm{Ppc}$ was significantly different from zero in all conditions (all $p<0.001$ ). Ppc amplitude did not vary as a function of distractor condition, $F_{(2,92)}=2.2, p=0.121, \eta^{2}=0.05$.

\section{Exploratory analysis: contralateral delay activity (CDA; 400-} $500 \mathrm{~ms}$ )

Visual inspection of the lateralized ERP waveforms showed that after typical analysis time windows of attentional components, a sustained negativity was found. A one-way ANOVA using each of the six conditions as a factor level indicated that CDA varied between conditions, $F_{(5,230)}=13.7, p<0.001, \eta^{2}=0.23$. Followup $t$ tests for dependent measures showed that the CDA elicited in trials with coinciding target and distractor positions was larger than in any other condition (all $p<0.001$ ), whereas the CDA did not vary among any other conditions (all $p \geq 0.169$ ), all twotailed.

\section{IEM results experiments 2 and 3}

Similar to ERP analyses, we collapsed the EEG data from experiments 2 and 3 to achieve higher statistical power. The CTFs are shown in Figure 5. As a measure of spatial selectivity, the slope for the CTFs (the rate of change in activity level from the central target position to further away locations) was calculated for both the target location (blue lines) and the distractor location (red lines). The IEM run on $\alpha$ bandpass $(8-12 \mathrm{~Hz}$ ) filtered data shows a slope reliably different from zero from 12 to $500 \mathrm{~ms}$ (targets) 

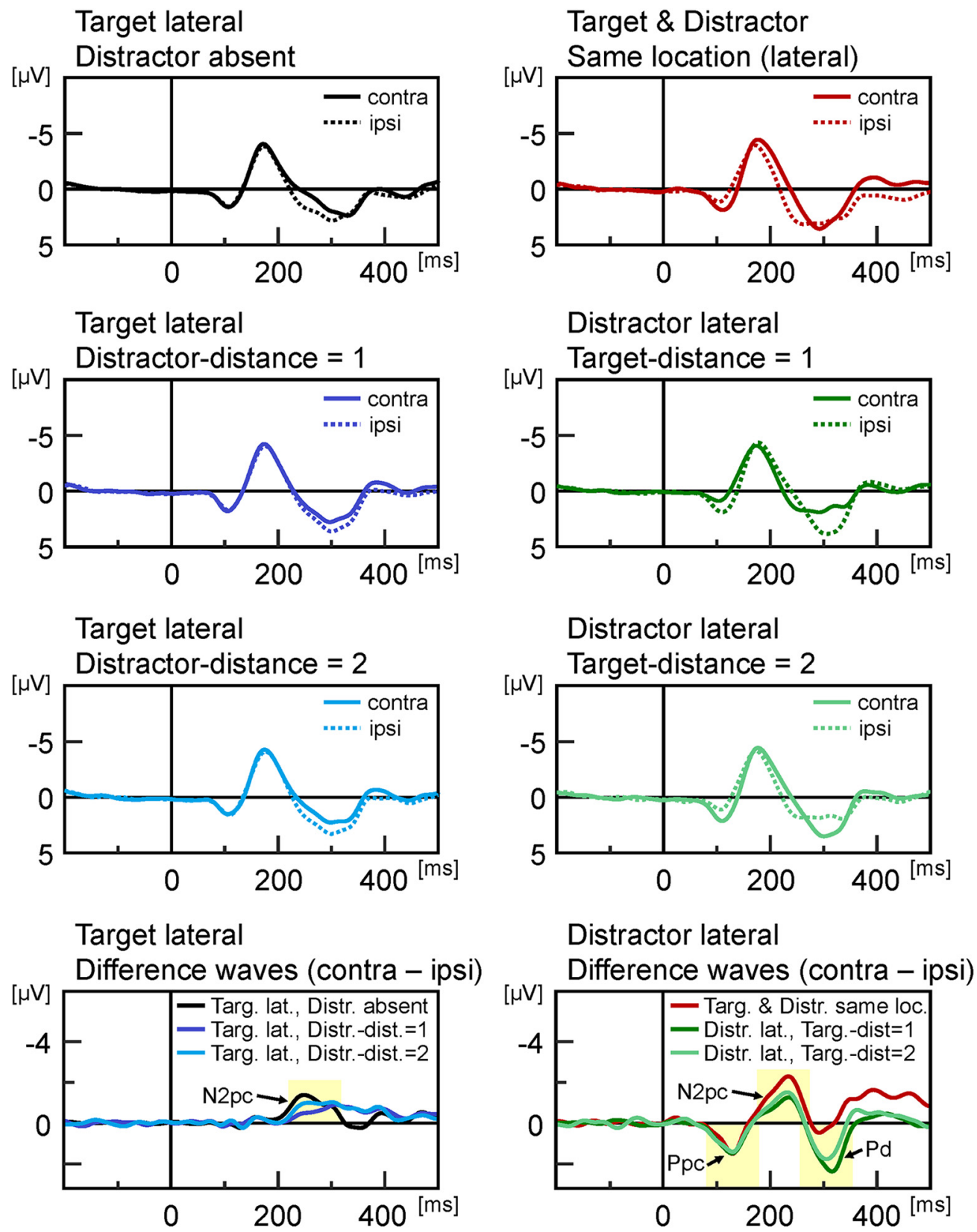

Figure 4. Grand average ERPs collapsed across experiments 2 and 3. The left panels show trials in which the target was presented laterally and the distractor was presented on the vertical midline or was absent (black line). The right panels show trials in which the distractor was presented laterally and the target was presented on the vertical midline (or target was at distractor location, red lines). The lowest row shows the difference waves (contra minus ipsilateral) for the same conditions as shown in the upper rows. Signal is pooled across P07/8, P7/8, and P03/4. For display purposes, signal was filtered with a 30-Hz lowpass filter. All statistical analyses were conducted on unfiltered data. Yellow shaded areas show the time windows used for statistical purposes.

and 32 to $289 \mathrm{~ms}$ (distractors). The distractor slope was reliably steeper than the target slope from 156 to $226 \mathrm{~ms}$. The target slope was reliably steeper than the distractor slope from 328 to $500 \mathrm{~ms}$. This shows that the channels were first selective for both target and distractor position, but more so for distractor position. Then, channels continued to be selective for the target position but not for the distractor position anymore.

The IEM run on $\theta$-bandpass $(4-8 \mathrm{~Hz})$ filtered data shows a slope reliably different from zero from 6 to $500 \mathrm{~ms}$ for the target stimulus; thus, the spatial distribution of activity in the $\theta$-band tracked the position of the target. $\theta$-Band activity also covaried with distractor position from 262 to $312 \mathrm{~ms}$, but in this case, the lowest level of channel activity was at the position of the distractor, such that there was an inverted tuning function surrounding the distractor position. Given that the target and distractor positions were completely balanced in this analysis, the inverted tuning function observed in the distractor analysis suggests a spatially-focused suppression of activity at the distractor position. Slopes for target and distractor were reliably different from 138 to $500 \mathrm{~ms}$.

\section{Discussion}

The present study documents a spatial gradient of suppression surrounding salient distractors while observers engaged in visual 
A $-3 \quad$ Alpha-band CTF: Target position

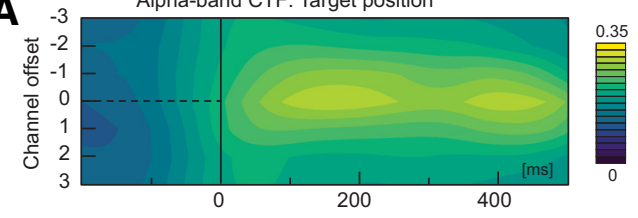

B $-3 \quad$ Alpha-band CTF: Distractor position
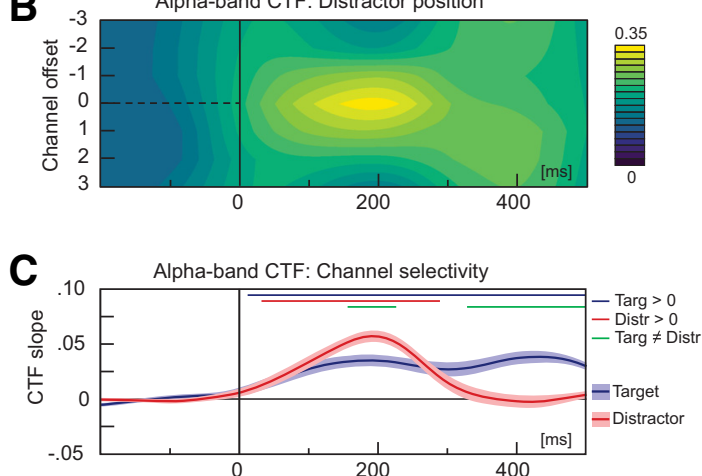

Theta-band CTF: Target position

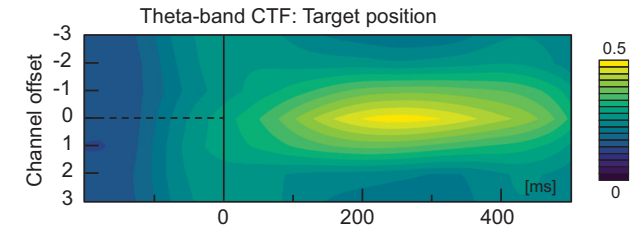

Theta-band CTF: Distractor position
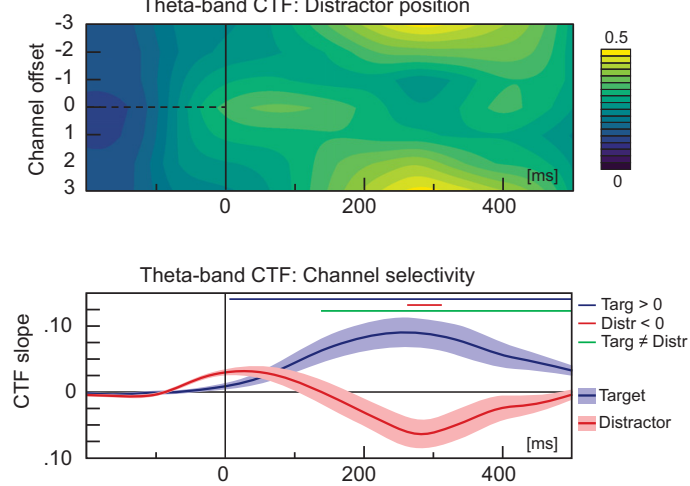

Figure 5. CTFs for evoked $\alpha$-band (8-12 Hz) activity (left panels) and evoked $\theta$-band (4-8 Hz) activity (right panels). All (TFs are trained on target position in distractor-absent trials and tested on $(\boldsymbol{A})$ target position and $(\boldsymbol{B})$ distractor positions in distractor-present trials. The slope of the (TFs in $(\boldsymbol{A})$ and $(\boldsymbol{B})$ is show in $(\boldsymbol{C})$ as blue lines (target) and red lines (distractor). Thin blue lines show time point where the target-CTF slope is different from zero, thin red lines show where the distractor-CTF is different from zero. Thin green lines show where target-CTF and distractor-CTF differ from one another. Only time points in clusters of at least 50 subsequent time points (= $50 \mathrm{~ms})$ with $p<0.05$ are highlighted. Shades indicate SEMs, corrected for individual differences (Cousineau, 2005).

search. We found that with decreasing distance between target and distractor in a visual search task, there were increases in both distractor interference and Pd amplitude, a neural marker of active suppression. Our results suggest that more suppression is applied when salient, irrelevant information is presented in close proximity to a target stimulus. At the same time, when target and distractor positions coincided and spatially directed suppression could interfere with target processing, the $\mathrm{Pd}$ was eliminated.

We used an additional singleton paradigm in which target and distractor location were independently positioned, allowing them to occupy the same location in a subset of trials (also referred to as "irrelevant singleton paradigm"; for review, see Becker, 2007). We replicated earlier findings showing that a salient distractor results in RT costs (Theeuwes, 1992, 2010; Feldmann-Wüstefeld et al., 2015; Sawaki and Luck, 2013). RT costs were most pronounced for singletons adjacent to the target and monotonically decreased to more distant locations, replicating earlier findings (Kwak et al., 1991; Caputo and Guerra, 1998; Mounts, 2000; Starreveld et al., 2004; Theeuwes et al., 2004; Koch et al., 2013; Gaspar and McDonald, 2014). This gradient of RT costs implies a spatial form of interference. Experiment 1a showed RT costs and distance effects when the target was the additional singleton at chance level (1/6 of trials), i.e., when there was no incentive for participants to attend red. Experiments $1 \mathrm{~b}$ and 2 replicated the results with compound stimuli, i.e., when an additional red ring was presented around the target in $1 / 6$ of the trials. Finally, experiment 3 showed the same pattern of results when the distractor color was determined by whether the distractor shared or did not share its location with the target, demonstrating that salient distractors interfere with target processing regardless of whether they are linked to relevant or irrelevant locations. Interestingly, all four experiments showed that in trials in which target and distractor shared the same location, RTs were shorter than for any other distance, but longer than for distractor-absent trials. This empirical pattern provides further evidence that some of the interference from singleton distractors is not explained by spatial capture alone.

Neural markers of attention provided further insight into how selection and suppression varied across these experimental conditions. We replicated prior work showing that the target$\mathrm{N} 2 \mathrm{pc}$ is smaller when target and distractor are in close proximity (Gaspar and McDonald, 2014), suggesting that attentional selection of relevant information may be impaired by nearby distractors. Using a systematic lateralization approach allowed us to disentangle specific neural signatures of target and distractor processing (Hickey et al., 2009) and scrutinize which attentional subprocesses can explain the spatial filtering costs observed in the behavioral data. Distractors elicited an N2pc, followed by a $\mathrm{Pd}$, suggesting they captured attention and were then suppressed (Burra and Kerzel, 2013; Feldmann-Wüstefeld and Schubö, 2013; Sawaki and Luck, 2013; Liesefeld et al., 2017). Importantly, the Pd component was larger for distractors adjacent to a target than for distractors further away from a target. Thus, both the Pd as a measure of distractor suppression and the N2pc as a measure of target enhancement were affected by target-distractor distance, but in an inverse fashion. This is in line with the neural ambiguity account that posits increased interference for stimuli that are presented in the same receptive field (Desimone and Duncan, 1995; Luck et al., 1997; Hickey and Theeuwes, 2011). Our results suggest that the visual system resolves neural ambiguity by applying more suppression to distractors near the target at the cost of enhancing the target itself. It was previously suggested that increased RT costs for distractors near targets are because of more attentional capture (Mounts, 2000; Hickey and Theeuwes, 2011). However, our results were inconsistent with this hypothesis, because the N2pc elicited by the distractor did not vary as a function of distance.

Although Pd increased with target-distractor proximity, no Pd was observed when they shared the same location, and instead a large N2pc was found. This suggests that target-distractor compounds were not suppressed but rather were strongly 
attended, in line with the notion that two coinciding singletons produce high activations on the priority map because of a redundancy gain (Krummenacher et al., 2002) and because of topdown and bottom-up factors dovetailing (Itti and Koch, 2001; Fecteau and Munoz, 2006; Wolfe, 2007). The Ppc that was associated with early suppression (Barras and Kerzel, 2017; Weaver et al., 2017; Feldmann-Wüstefeld and Vogel, 2019) was not affected by target-distractor distance. This may be because the Ppc reflects an early "attend-to-me" signal (Sawaki and Luck, 2010) that is not modulated by the amount of interference but rather reflects activity on the priority/salience map (Barras and Kerzel, 2017). In this context the Pd can be seen as a response to that signal that reflects increasing levels of suppression of the distractor as interference from the distractor increases. The relative timing of N2pc and Pd allow an approximation of the time course of attentional processes. In line with earlier studies (Hilimire et al., 2012; Feldmann-Wüstefeld and Schubö, 2013; Feldmann-Wüstefeld et al., 2015; Liesefeld et al., 2017), attention was first captured by the most salient item, then the most salient item was suppressed while the target was attended. Apparently, participants did not proactively suppress the distractors as was shown in previous studies (Gaspelin et al., 2015; see also Gaspelin and Luck, 2018; Feldmann-Wüstefeld et al., 2020). This may have been because of the occasional co-occurrence of target and distractor which could de-incentivize the suppression of distractors before localizing the target.

The IEM allowed us to examine spatially selective EEG activity that tracked the position of both targets and distractors. $\alpha$-Band $(8-12 \mathrm{~Hz})$ activity showed that initially, both targets and distractors were attended, but distractors more so than targets. The color contrast was strong for the distractor singletons, such that they were likely more salient than the shape-defined target singletons. Thus, stronger initial capture by the distractor falls in line with the notion that salient stimuli capture attention automatically and may receive priority over less salient, yet task-relevant items (Theeuwes, 2010; see also Wyble et al., 2020). The spatial selectivity for distractor positions was transient, however, and later exceeded by the spatial selectivity of targets (from $\sim 280 \mathrm{~ms}$ on, only targets were attended), in line with the idea that attentional capture is followed by rapid disengagement (Belopolsky et al., 2010) as well as with the idea that topdown attention deployment eventually overrides attentional capture and thus supports encoding of task-relevant information (Leber and Egeth, 2006). We also show that $\theta$-band and $\alpha$-band activity are picking up on qualitatively different aspects of attentional processing. A previous study used the lateralized $\theta$-band activity to measure attention deployment and found that $\theta$ tracked both target and distractor activity (Harris et al., 2017). In line with this, the present study showed that by means of an IEM, $\theta$-band $(4-8 \mathrm{~Hz})$ activity tracked the position of the target as well as the distractor. The CTF for the distractor, however, was inverted, suggesting spatial suppression of the irrelevant item. The IEM showed highest spatial selectivity for the target and a monotonic fall in channel activity as distance from that position increased. Importantly, for the distractor it showed belowbaseline spatial selectivity, most pronounced at $\sim 290 \mathrm{~ms}$. The lowest channel activity in the $\theta$-band was observed at the position of the distractor, with a monotonic rise in channel activity as distance from that position increased. Thus, spatially selective activity in the $\theta$-band reveals a relative suppression of channel activity at distractor positions, indicating a spatially-directed form of distractor suppression. As the channel activity for any given distractor location is based on equally many trials with targets at all six locations (including the distractor location), the below-baseline activity cannot be explained with more pronounced activity induced by targets at non-distractor locations. It should be noted that the spatial selectivity of the IEM reflects activity phase-locked to stimulus onset because it is based on the evoked EEG signal. As $\alpha$ and $\theta$ are strongly reflected in the ERP (Sauseng et al., 2007; Harris et al., 2017), it is thus possible that the IEM taps into processes that overlap with those that elicit ERP components such as the N2pc and Pd. That said, the IEM provided a fine-grained measure of spatial selectivity that would not have been possible with ERP components alone (six positions rather than two hemifields, Fahrenfort et al., 2017).

\section{Spatial and nonspatial interference}

The increasing RT costs with decreasing target-distractor distance indicate that distractors induced spatial interference. The graded Pd and target-N2pc amplitude as well as the graded tuning profile of $\alpha$-band and $\theta$-band activity further support this notion. Analogously, the reduced RT costs and the larger target$\mathrm{N} 2 \mathrm{pc}$ for targets and distractors sharing the same location compared with Dist $>0$ trials indicate a spatial benefit. We also found compatibility effects: RTs were faster when target and distractor identity were identical, indicative of spatial attention deployment toward the distractor (Folk and Remington, 2006; Becker, 2007). At the same time, distractor-N2pc amplitudes were distance-invariant, demonstrating that distractors did not capture attention more when they were near targets. On the contrary, when distractor and target coincided, the N2pc was larger than for distractors or targets presented alone. Interestingly, performance was worse in this condition than when no distractor was presented at all (distance-zero trials), although a pure spatial capture account (Theeuwes, 1992, 2010) would predict that spatially coincident targets and distractors should elicit robust spatial capture and thus produce the shortest RTs. Thus, distractor interference for distance-zero trials suggests that distractors also induced non-spatial filtering costs, in line with prior demonstrations (Folk and Remington, 1998, 2006; Becker, 2007; Wykowska and Schubö, 2011). Same location costs were previously observed in contingent capture paradigms in which a salient distractor was occasionally presented at the upcoming target position (Lamy et al., 2004; Eimer et al., 2009; Carmel and Lamy, 2014; Schoeberl et al., 2018). One possibility is that the target and distractor compete for encoding resources, such that the extraction of target features is delayed even when target and distractor are presented in the same location (Kahneman et al., 1983; Treisman et al., 1983; Folk and Remington, 2006). The larger CDA amplitude we found in trials with a coinciding target and distractor may be the neural correlate of such increased competition.

In sum, we used ERPs and an IEM to demonstrate that the visual system responds to distractor interference with a spatial gradient of suppression: the $\mathrm{Pd}$ component in the ERP increased with increasing proximity of target and distractor while the theta-band IEM showed most suppressed activity at the distractor location and monotonically decreasing suppression toward more distant locations. Whereas suppression is strongest for distractors presented near the target, selection is weakest for targets presented near a distractor. Thus our results provide robust evidence for a spatially directed process for 
suppressing distractor interference during goal-driven visual search.

\section{References}

Awh E, Belopolsky AV, Theeuwes J (2012) Top-down versus bottom-up attentional control: a failed theoretical dichotomy. Trends Cogn Sci 16:437-443.

Barras C, Kerzel D (2017) Salient-but-irrelevant stimuli cause attentional capture in difficult, but attentional suppression in easy visual search. Psychophysiology 54:1826-1838.

Becker SI (2007) Irrelevant singletons in pop-out search: attentional capture or filtering costs? J Exp Psychol Hum Percept Perform 33:764-787.

Belopolsky AV, Schreij D, Theeuwes J (2010) What is top-down about contingent capture? Atten Percept Psychophys 72:326-341.

Burra N, Kerzel D (2013) Attentional capture during visual search is attenuated by target predictability: evidence from the N2pc, Pd, and topographic segmentation. Psychophysiology 50:422-430.

Burra N, Kerzel D (2014) The distractor positivity (Pd) signals lowering of attentional priority: evidence from event-related potentials and individual differences. Psychophysiology 51:685-696.

Caputo G, Guerra S (1998) Attentional selection by distractor suppression. Vision Res 38:669-689.

Carmel T, Lamy D (2014) The same-location cost is unrelated to attentional settings: an object-updating account. J Exp Psychol Hum Percept Perform 40:1465-1478.

Chang S, Egeth HE (2019) Enhancement and suppression flexibly guide attention. Psychol Sci 30:1724-1732.

Cousineau D (2005) Confidence intervals in within-subject designs: a simpler solution to Loftus and Masson's method. Tutor Quant Methods Psychol $1: 42-45$.

Desimone R, Duncan J (1995) Neural mechanisms of selective visual attention. Annu Rev Neurosci 18:193-222.

Dowdall JR, Luczak A, Tata MS (2012) Temporal variability of the N2pc during efficient and inefficient visual search. Neuropsychologia 50:2442-2453.

Eimer M (1996) The N2pc component as an indicator of attentional selectivity. Electroencephalogr Clin Neurophsiol 99:225-234.

Eimer M, Kiss M, Press C, Sauter D (2009) The roles of feature-specific task set and bottom-up salience in attentional capture: an ERP study. J Exp Psychol Hum Percept Perform 35:1316-1328.

Ester EF, Sprague TC, Serences JT (2015) Parietal and frontal cortex encode stimulus-specific mnemonic representations during visual working memory. Neuron 87:893-905.

Fahrenfort JJ, Grubert A, Olivers CNL, Eimer M (2017) Multivariate EEG analyses support high-resolution tracking of feature-based attentional selection. Sie Rep 7:1886.

Fecteau J, Munoz D (2006) Salience, relevance, and firing: a priority map for target selection. Trends Cogn Sci 10:382-390.

Feldmann-Wüstefeld T, Schubö A (2013) Context homogeneity facilitates both distractor inhibition and target enhancement. J Vis 13:11.

Feldmann-Wüstefeld T, Uengoer M, Schubö A (2015) You see what you have learned. Evidence for an interrelation of associative learning and visual selective attention. Psychophysiology 52:1483-1497.

Feldmann-Wüstefeld T, Vogel EK (2019) Neural evidence for the contribution of active suppression during working memory filtering. Cereb Cortex 29:529-543.

Feldmann-Wüstefeld T, Awh E (2020) Alpha-band activity tracks the zoom lens of attention. J Cogn Neurosci 32:272-282.

Feldmann-Wüstefeld T, Busch NA, Schubö A (2020) Failed suppression of salient stimuli precedes behavioral errors. J Cogn Neurosci 32:367-377.

Fockert JD, Rees G, Frith C, Lavie N (2004) Neural correlates of attentional capture in visual search. J Cogn Neurosci 16:751-759.

Folk CL, Remington RW (1998) Selectivity in distraction by irrelevant featural singletons: evidence for two forms of attentional capture. J Exp Psychol Hum Percept Perform 24:847-858.

Folk CL, Remington R (2006) Top-down modulation of preattentive processing: testing the recovery account of contingent capture. Vis Cogni 14:445-465.

Foster JJ, Bsales EM, Jaffe RJ, Awh E (2017) Alpha-band activity reveals spontaneous representations of spatial position in visual working memory. Current Biology 27:3216-3223.
Foster JJ, Sutterer DW, Serences JT, Vogel EK, Awh E (2016) The topography of alpha-band activity tracks the content of spatial working memory. J Neurophysiol 115:168-177.

Gaspar JM, Mcdonald JJ (2014) Suppression of salient objects prevents distraction in visual search. J Neurosci 34:5658-5666.

Gaspelin N, Luck SJ (2018) Combined electrophysiological and behavioral evidence for the suppression of salient distractors. J Cogn Neurosci 30:1265-1280.

Gaspelin N, Leonard CJ, Luck SJ (2015) Direct evidence for active suppression of salient-but-irrelevant sensory inputs. Psychol Sci 26:1740-1750.

Harris AM, Dux PE, Jones CN, Mattingley JB (2017) Distinct roles of theta and alpha oscillations in the involuntary capture of goal-directed attention. Neuroimage 152:171-183.

Hickey C, Theeuwes J (2011) Context and competition in the capture of visual attention. Atten Percept Psychophys 73:2053-2064.

Hickey C, Di Lollo V, McDonald JJ (2009) Electrophysiological indices of target and distractor processing in visual search. J Cogn Neurosci 21:760-775.

Hilimire MR, Hickey C, Corballis PM (2012) Target resolution in visual search involves the direct suppression of distractors: evidence from electrophysiology. Psychophysiology 49:504-509.

Hodsoll J, Mevorach C, Humphreys GW (2009) Driven to less distraction: RTMS of the right parietal cortex reduces attentional capture in visual search. Cereb Cortex 19:106-114.

Hutchinson JB, Turk-Browne NB (2012) Memory-guided attention: Control from multiple memory systems. Trends in cognitive sciences 16:576-579.

Itti L, Koch C (2001) Computational modeling of visual attention. Nat Rev Neurosci 2:1-11.

Kahneman D, Treisman A, Burkell J (1983) The cost of visual filtering. J Exp Psychol Hum Percept Perform 9:510-522.

Koch AI, Müller HJ, Zehetleitner M (2013) Distractors less salient than targets capture attention rather than producing non-spatial filtering costs. Acta Psychol (Amst) 144:61-72.

Krummenacher J, Müller HJ, Heller D (2002) Visual search for dimensionally redundant pop-out targets: parallel-coactive processing of dimensions is location specific. J Exp Psychol Hum Percept Perform 28:1303-1322.

Kwak HW, Dagenbach D, Egeth H (1991) Further evidence for a timeindependent shift of the focus of attention. Percept Psychophys 49:473-480.

Lamy D, Leber A, Egeth HE (2004) Effects of task relevance and stimulusdriven salience in feature-search mode. J Exp Psychol Hum Percept Perform 30:1019-1031

Leber AB, Egeth HE (2006) It's under control: top-down search strategies can override attentional capture. Psychon Bull Rev 13:132-138.

Liesefeld HR, Müller HJ (2019) Distractor handling via dimension weighting. Curr Opin Psychol 29:160-167.

Liesefeld HR, Liesefeld AM, Töllner T, Müller HJ (2017) Attentional capture in visual search: capture and post-capture dynamics revealed by EEG. Neuroimage 156:166-173.

Luck SJ, Hillyard SA (1994) Spatial filtering during visual search: evidence from human electrophysiology. J Exp Psychol Hum Percept Perform 20:1000-1014

Luck SJ, Girelli M, McDermott MT, Ford MA (1997) Bridging the gap between monkey neurophysiology and human perception: an ambiguity resolution theory of visual selective attention. Cogn Psychol 33:64-87.

Mounts JRW (2000) Attentional capture by abrupt onsets and feature singletons produces inhibitory surrounds. Percept Psychophys 62:1485-1493

Papaioannou O, Luck SJ (2020) Effects of eccentricity on the attentionrelated N2pc component of the event-related potential waveform. Psychophysiology 57:e13532.

Sauseng P, Klimesch W, Stadler W, Schabus M, Doppelmayr M, Hanslmayr S, Gruber WR, Birbaumer N (2005) A shift of visual spatial attention is selectively associated with human EEG alpha activity. Eur J Neurosci 22:2917-2926.

Sauseng P, Klimesch W, Gruber WR, Hanslmayr S, Freunberger R, Doppelmayr M (2007) Are event-related potential components generated by phase resetting of brain oscillations? A critical discussion. Neuroscience 146:1435-1444.

Sawaki R, Luck SJ (2010) Capture versus suppression of attention by salient singletons: electrophysiological evidence for an automatic attend-to-me signal. Atten Percept Psychophys 72:1455-1470. 
Sawaki R, Luck SJ (2013) Active suppression after involuntary capture of attention. Psychon Bull Rev 20:296-301.

Schoeberl T, Ditye T, Ansorge U (2018) Same-location costs in peripheral cueing: the role of cue awareness and feature changes. J Exp Psychol Hum Percept Perform 44:433-451.

Starreveld PA, Theeuwes J, Mortier K (2004) Response selection in visual search: the influence of response compatibility of nontargets. J Exp Psychol Hum Percept Perform 30:56-78.

Theeuwes J (1992) Perceptual selectivity for color and form. Percept Psychophys 51:599-606.

Theeuwes J (2010) Top-down and bottom-up control of visual selection. Acta Psychol (Amst) 135:77-99.

Theeuwes J, Kramer AF, Kingstone A (2004) Attentional capture modulates perceptual sensitivity. Psychon Bull Rev 11:551-554.

Treisman A, Kahneman D, Burkell J (1983) Perceptual objects and the cost of filtering. Percept Psychophys 33:527-532.
Weaver MD, van Zoest W, Hickey C (2017) A temporal dependency account of attentional inhibition in oculomotor control. Neuroimage 147:880894

Wolfe JM (2007) Guided search 4.0: current progress with a model of visual search. In: Integrated models of cognitive systems, pp 99-119. Oxford: Oxford University Press.

Woodman GF, Luck SJ (2003) Serial deployment of attention during visual search. J Exp Psychol Hum Percept Perform 29:121-138.

Wyble B, Callahan-Flintoft C, Chen H, Marinov T, Sarkar A, Bowman H (2020) Understanding visual attention with RAGNAROC: a reflexive attention gradient through neural AttRactOr competition. Psychol Rev 127:1163-1198.

Wykowska A, Schubö A (2011) Irrelevant singletons in visual search do not capture attention but can produce nonspatial filtering costs. J Cogn Neurosci 23:645-660.

Yantis S, Egeth HE (1999) On the distinction between visual salience and stimulusdriven attentional capture. J Exp Psychol Hum Percept Perform 25:661-676. 